\title{
Oncologic Outcomes of Immediate Breast Reconstruction in Young Women with Breast Cancer Receiving Neoadjuvant Chemotherapy
}

Zhen-Yu Wu ( $\nabla$ wuzyps@gmail.com )

Asan Medical Center https://orcid.org/0000-0003-1731-6370

Hee Jeong Kim

Asan Medical Center

Jongwon Lee

Asan Medical Center

II Yong Chung

Asan Medical Center

Jisun Kim

Asan Medical Center

Sae byul Lee

Asan Medical Center

Byung-Ho Son

Asan Medical Center

Eun key Kim

Asan Medical Center

Jae Ho Jeong

Asan Medical Center

Hee Jin Lee

Asan Medical Center

Eun Young Chae

Asan Medical Center

Jinhong Jung

Asan Medical Center

Sei-Hyun Ahn

Asan Medical Center

Beom Seok Ko

Asan Medical Center 
Keywords: Breast cancer, young age, immediate breast reconstruction, neoadjuvant chemotherapy, oncologic safety

Posted Date: July 7th, 2021

DOI: https://doi.org/10.21203/rs.3.rs-673315/v1

License: (c) (1) This work is licensed under a Creative Commons Attribution 4.0 International License. Read Full License

Version of Record: A version of this preprint was published at Breast Cancer Research and Treatment on October 31st, 2021. See the published version at https://doi.org/10.1007/s10549-021-06428-9. 


\section{Abstract}

Background: Oncologic safety of postmastectomy breast reconstruction in young women with breast cancer is not well-defined, especially in the setting of neoadjuvant chemotherapy (NACT). We retrospectively compared the oncologic outcomes following nipple-sparing (NSM)/skin-sparing mastectomy (SSM) with immediate breast reconstruction (IBR) and conventional mastectomy (CM) alone in young breast cancer patients after NACT.

Methods: A total of 1266 women with primary breast cancer who underwent NACT followed by total mastectomy with or without IBR were reviewed. Of these, only young patients (age $\leq 40$ years at diagnosis) were included in the outcome analysis $(n=375)$. After propensity score-matching by clinical $T$ and $\mathrm{N}$ stage, molecular subtype, response to NACT, and adjuvant radiotherapy status, 228 patients were 1:1 matched, comprising balanced IBR group (with NSM/SSM) and CM-alone group.

Results: The 5-year locoregional recurrence-free, disease-free, distant metastasis (DM)-free, and breast cancer-specific survival (BCSS) rates for the entire cohort of young patients were $83.4 \%, 65.3 \%, 71.7 \%$, and $85.4 \%$, respectively. Locoregional recurrence rates between the matched groups were similar ( $14 \%$ vs. $15.8 \% ; P=0.710)$; however, IBR group had significantly lower DM rate $(27.2 \%$ vs. $40.4 \% ; P=0.036)$ and breast cancer mortality $(14.9 \%$ vs. $27.2 \% ; P=0.023)$ than $\mathrm{CM}$-alone group. IBR group showed significantly improved 5-year DM-free survival $(74.1 \%$ vs. $62.6 \% ; P=0.043)$ and BCSS $(89.1 \%$ vs. $77.6 \% ; P$ $=0.048$ ) rates than CM-alone group.

Conclusions: Our results indicated that IBR with NSM/SSM does not negatively affect long-term oncologic outcomes compared to $\mathrm{CM}$ alone in young women with breast cancer receiving NACT.

\section{Introduction}

The incidence of breast cancer is increasing worldwide [1]. According to the data from GLOBOCAN, approximately 2.2 million women have been newly diagnosed with breast cancer in 2020, accounting for $24.5 \%$ of all female malignancies [1]. The proportion of young patients, which generally refers to patients under 40 years of age, among all breast cancer patients is not large $(4-7 \%$ in Western countries $[2,3]$ and $10-14 \%$ in Asian countries [4,5]); however, breast cancer is still the most prevalent cancer with the highest mortality among young women [6]. Historically, young age at diagnosis itself is a prognostic factor associated with adverse breast cancer outcomes [7]. Compared to the older counterparts, younger women with breast cancer are more likely to present with more aggressive tumor characteristics such as higher tumor grade, larger tumor size, higher incidence of nodal involvement, and more lympho-vascular invasion (LVI) [8-10]. In addition, because of the higher likelihood of having hormone receptor-negative tumors [8-10], young patients are more likely to benefit from neoadjuvant chemotherapy (NACT).

In addition to the concerns regarding cancer diagnosis, young women with breast cancer may also be distressed at facing the potential oncologic treatment-associated damage to their body image [11]. Although breast-conserving surgery may have less negative impact on the patient's postoperative body 
image and quality of life than other surgical procedures [12] and NACT can increase the rate of breast conservation [13], there is still a large proportion of patients who will undergo total mastectomy after receiving NACT $[14,15]$. Nipple-sparing (NSM) or skin-sparing mastectomy (SSM), combined with immediate breast reconstruction (IBR), can be an important alternative for young women with breast cancer because this procedure can provide superior aesthetic results and improved patients' quality of life compared with conventional mastectomy (CM) alone [16-18]. Several studies have documented increasing trends in NSM/SSM and breast reconstruction in younger patients [19-21]. However, the oncologic safety of this procedure is not well-defined in young women with breast cancer, especially in the setting of NACT. To our knowledge, to date, no study has compared the oncologic outcomes of IBR and $\mathrm{CM}$ alone in young breast cancer patients receiving NACT. Therefore, in this study, we sought to compare the oncologic outcomes following IBR and CM alone in young patients with breast cancer who were treated with NACT.

\section{Patients And Methods}

The records from a prospectively maintained database were reviewed for patients who had undergone NACT followed by total mastectomy, with or without immediate breast reconstruction, between January 1 , 2010, and November 30, 2016 at Asan Medical Center (Seoul, Korea) after receiving institutional review board approval. We excluded patients with inflammatory breast cancer or pathological T4 disease, synchronous distant metastasis (DM), or recurrent disease. Only patients aged $\leq 40$ years at the time of diagnosis were included in the outcome analysis.

All patients included in this study received NACT after the diagnosis of breast cancer. The NACT regimens were selected at the discretion of the treating oncologist. All patients who underwent IBR were combined with NSM or SSM. NSM/SSM was performed according to the indications of conventional mastectomy, as long as there was no evidence for tumor involvement in the breast skin/nipple-areolar complex clinically and on imaging. In NSM cases, retroareolar frozen-section biopsy specimens were collected and examined intraoperatively. The nipple-areola complex was preserved if the shape, color, and palpated features of the nipple were normal, and if the nipple margin was confirmed to be tumor-free on frozensection biopsy. In cases in which the retroareolar tissue was positive for malignancy in the frozen section or permanent biopsy, the nipple with or without the areola was removed, and the surgical procedure was converted to SSM. In our entire cohort, no patient was converted to CM due to failure of the NSM/SSM procedure.

Postoperatively, the patients were regularly followed up every 3-6 months for the first 5 years and annually thereafter. Recurrences and metastases were assessed by clinical examination and chest radiography during every follow-up visit. Abnormal clinical findings have been evaluated through further studies, including ultrasonography, computed tomography of the chest, and bone scanning. In patients suspected of having a local or regional recurrence, fine needle aspiration, core-needle, or excisional biopsy was performed for pathological confirmation. In patients diagnosed with locoregional recurrence (LRR), we generally performed positron emission tomography (PET)-computed tomography (CT) to rule out 
DMs. However, in some cases, CT and/or bone scan was performed instead of PET-CT based on the judgment of the treating physician. Identification of lesions with clear evidence of distant metastasis on imaging was considered recurrence without pathological examination. LRRs were classified as biopsyproven local recurrences in the ipsilateral nipple-areola complex, skin/subcutaneous layer, or chest wall and as regional recurrences defined as carcinoma metastases involving the ipsilateral axillary, supraclavicular, or internal mammary lymph node. Any other site of recurrence was considered to be DM.

To reduce the effects of selection bias on the type of surgery and confounding factors on the comparison of the oncologic outcomes between the IBR and CM-alone groups, we used propensity-score matching to create well-balanced groups. We included the following baseline covariates for matching: clinical T stage, clinical $\mathrm{N}$ stage, molecular subtype, pathological complete response (pCR) status, and adjuvant radiotherapy status. Propensity scores were calculated for each patient using a multivariate logistic regression model, and patients were matched 1:1 into IBR with NSM/SSM and CM-alone groups using caliper restriction to the nearest neighbor without replacement. Tumor stage was determined according to the 8th edition of the American Joint Committee on Cancer Staging Manual [22]. A pCR was defined as no evidence of invasive cancer in the breast and axillary lymph node.

\section{Statistical Analyses}

The endpoints of interest were locoregional recurrence-free survival (LRFS), disease-free survival (DFS), distant metastasis-free survival (DMFS), and breast cancer-specific survival (BCSS). Patients with initial DM were excluded from the LRR group. In cases of concurrent LRR or DM, each recurrence was counted simultaneously as an event. Follow-up was calculated from the date of diagnosis. The LRFS, DFS, DMFS, and BCSS rates were calculated using the Kaplan-Meier method and compared using log-rank tests. The chi-squared or Fisher's exact test was used for intergroup comparisons. The clinicopathological factors associated with DMFS were analyzed using the univariate and multivariate Cox proportional hazards regression model. All statistical analyses were performed using the International Business Machines Corporation (IBM) Statistical Package for the Social Sciences Statistics for Windows version 24.0 (IBM Corp., Armonk, NY, USA). Two-tailed $P$-values $<0.05$ were considered statistically significant. Data analysis was performed from August 1, 2020, to February 26, 2021.

\section{Results}

Baseline Characteristics for the Entire Cohort

A total of 1395 patients were treated with NACT and mastectomy during the study period. Among these, patients with inflammatory breast cancer or pathological T4 disease, synchronous DM, or recurrent disease were excluded $(n=129)$. Accordingly, 1266 patients were included in the analysis. Among these, 375 patients $(29.6 \%)$ were aged $\leq 40$ years, and 891 patients $(70.4 \%)$ were aged $>40$ years. IBR was performed in $64.3 \%$ of patients aged $\leq 40$ years and in $32 \%$ of those aged $>40$ years $(P<0.001)$. The baseline characteristics for the 375 patients aged $\leq 40$ years are shown in Table 1 . The study cohort 
comprised 241 patients who underwent IBR with NSM/SSM and 134 patients who underwent CM alone. Patients in the IBR group were more likely to be at earlier clinical and pathological stages, to have hormone-positive disease, and to receive adjuvant radiotherapy than those in the $\mathrm{CM}$-alone group. However, the pCR status did not differ significantly between the groups. 
Table 1

Baseline characteristic for the entire cohort before matching

\begin{tabular}{|c|c|c|c|c|c|c|c|c|}
\hline \multicolumn{2}{|l|}{ Variables } & \multirow{2}{*}{$\begin{array}{l}\text { Total, } \mathbf{n} \\
=\mathbf{3 7 5} \\
36(23- \\
40)\end{array}$} & \multirow[t]{2}{*}{$\%$} & \multirow{2}{*}{$\begin{array}{l}\text { IBR, } \mathbf{n} \\
=\mathbf{2 4 1} \\
36 \\
(23- \\
40)\end{array}$} & \multirow[t]{2}{*}{$\%$} & \multirow{2}{*}{$\begin{array}{l}\text { CM, n } \\
=134 \\
37 \\
(25- \\
40)\end{array}$} & \multirow[t]{2}{*}{$\%$} & \multirow[t]{2}{*}{$P$} \\
\hline $\begin{array}{l}\text { Age, Median } \\
\text { (range), years }\end{array}$ & & & & & & & & \\
\hline \multirow[t]{3}{*}{ Clinical T stage } & cT1 & 18 & 4.8 & 17 & 7.1 & 1 & 0.7 & $\begin{array}{l}< \\
0.001\end{array}$ \\
\hline & cT2 & 185 & 49.3 & 130 & 53.9 & 55 & 41.0 & \\
\hline & cT3-4 & 172 & 45.9 & 94 & 39.0 & 78 & 58.2 & \\
\hline \multirow[t]{3}{*}{ Clinical N stage } & $\mathrm{cNO}$ & 98 & 26.1 & 76 & 31.5 & 22 & 16.4 & $\begin{array}{l}<.001 \\
0.00\end{array}$ \\
\hline & $\mathrm{cN} 1$ & 176 & 46.9 & 121 & 50.2 & 55 & 41.0 & \\
\hline & cN2-3 & 101 & 26.9 & 44 & 18.3 & 57 & 42.5 & \\
\hline \multirow[t]{4}{*}{ Molecular subtype } & HR+/HER2- & 171 & 45.6 & 122 & 50.6 & 49 & 36.6 & 0.001 \\
\hline & $\mathrm{HR}+/ \mathrm{HER} 2+$ & 93 & 24.8 & 64 & 26.6 & 29 & 21.6 & \\
\hline & HR-/HER2+ & 46 & 12.3 & 26 & 10.8 & 20 & 14.9 & \\
\hline & TNBC & 65 & 17.3 & 29 & 12.0 & 36 & 26.9 & \\
\hline \multirow[t]{2}{*}{$\mathrm{pCR}$} & Yes & 43 & 11.5 & 29 & 12.0 & 14 & 10.4 & 0.644 \\
\hline & No & 332 & 88.5 & 212 & 88.0 & 120 & 89.6 & \\
\hline \multirow{3}{*}{$\begin{array}{l}\text { Pathological T } \\
\text { stage }\end{array}$} & урт0/Tis & 51 & 13.6 & 32 & 13.3 & 19 & 14.2 & 0.236 \\
\hline & урT1 & 127 & 33.9 & 89 & 36.9 & 38 & 28.4 & \\
\hline & урТ2-3 & 197 & 52.5 & 120 & 49.8 & 77 & 57.5 & \\
\hline \multirow{3}{*}{$\begin{array}{l}\text { Pathological } N \\
\text { stage }\end{array}$} & ypNO & 149 & 39.7 & 102 & 42.3 & 47 & 35.1 & 0.146 \\
\hline & ypN1 & 140 & 37.3 & 91 & 37.8 & 49 & 36.6 & \\
\hline & ypN2-3 & 86 & 22.9 & 48 & 19.9 & 38 & 28.4 & \\
\hline \multirow[t]{2}{*}{ Histotype } & IDC & 329 & 87.7 & 214 & 88.8 & 115 & 85.8 & 0.461 \\
\hline & ILC & 7 & 1.9 & 4 & 1.7 & 3 & 2.2 & \\
\hline
\end{tabular}

$\mathrm{CM}$, conventional mastectomy; HER2, human epidermal growth factor receptor 2; HR, hormone receptor; IBR, immediate breast reconstruction; IDC, invasive ductal carcinoma; ILC, invasive lobular carcinoma; LVI, lymphovascular invasion; NACT, neoadjuvant chemotherapy; pCR, pathological complete response; TNBC, triple-negative breast cancer. *Unknown, $\mathrm{n}=37$ 


\begin{tabular}{|c|c|c|c|c|c|c|c|c|}
\hline \multicolumn{2}{|l|}{ Variables } & \multirow{2}{*}{$\begin{array}{l}\text { Total, } \mathrm{n} \\
=375 \\
22\end{array}$} & \multirow{2}{*}{$\%$} & \multirow{2}{*}{$\begin{array}{l}\text { IBR, } \mathrm{n} \\
=241 \\
15\end{array}$} & \multirow{2}{*}{$\%$} & \multirow{2}{*}{$\begin{array}{l}\text { CM, n } \\
=134 \\
7\end{array}$} & \multirow{2}{*}{$\%$} & \multirow[t]{2}{*}{$P$} \\
\hline & Mixed & & & & & & & \\
\hline & Others & 17 & 4.5 & 8 & 3.3 & 9 & 6.7 & \\
\hline \multirow[t]{2}{*}{ Histologic grade } & 1,2 & 245 & 65.3 & 162 & 67.2 & 83 & 61.9 & 0.303 \\
\hline & 3 & 130 & 34.7 & 79 & 32.8 & 51 & 38.1 & \\
\hline \multirow[t]{2}{*}{ LVI } & Yes & 162 & 43.2 & 107 & 44.4 & 55 & 41.0 & 0.53 \\
\hline & No & 213 & 56.8 & 134 & 55.6 & 79 & 59.0 & \\
\hline \multirow[t]{2}{*}{ Post-NACT Ki67 * } & $<10 \%$ & 154 & 45.6 & 103 & 48.1 & 51 & 41.1 & 0.213 \\
\hline & $\geq 10 \%$ & 184 & 54.4 & 111 & 51.9 & 73 & 58.9 & \\
\hline \multirow[t]{2}{*}{$\begin{array}{l}\text { Adjuvant } \\
\text { radiotherapy }\end{array}$} & Yes & 237 & 63.2 & 130 & 53.9 & 107 & 79.9 & $\begin{array}{l}< \\
0.001\end{array}$ \\
\hline & No & 138 & 36.8 & 111 & 46.1 & 27 & 20.1 & \\
\hline \multicolumn{9}{|c|}{$\begin{array}{l}\mathrm{CM} \text {, conventional mastectomy; HER2, human epidermal growth factor receptor } 2 \text {; HR, hormone } \\
\text { receptor; IBR, immediate breast reconstruction; IDC, invasive ductal carcinoma; ILC, invasive lobular } \\
\text { carcinoma; LVI, Iymphovascular invasion; NACT, neoadjuvant chemotherapy; pCR, pathological } \\
\text { complete response; TNBC, triple-negative breast cancer. *Unknown, } n=37\end{array}$} \\
\hline
\end{tabular}

Oncologic Outcomes of the Entire Cohort

The median follow-up duration for the entire cohort $(n=375)$ was 72 (range, 9-138) months. During this period, recurrences were observed in 137 patients (36.5\%). DM was observed in 112 patients (29.9\%); LRR as the first event was observed in 60 patients (16\%). Breast cancer-associated death occurred in 70 patients (18.7\%). The median time to DM and LRR as the first event was 30 months (range, 6-99 months) and 24 months (range, 5-76 months), respectively. The 5-year LRFS, DFS, DMFS, and BCSS rates were $83.4 \%, 65.3 \%, 71.7 \%$, and $85.4 \%$, respectively. A total of 43 patients $(11.5 \%)$ achieved pCR. Among these, 6 patients (14\%) showed recurrence, including 3 patients $(7.0 \%)$ with DM, 2 patients $(4.7 \%)$ with LRR, and 1 patient (2.3\%) with concurrent LRR and DM. The following factors were significantly associated with reduced DMFS in the univariate analysis: clinical $\mathrm{N}$ stage, pathological T stage, pathological nodal status, triple-negative subtype, pCR status, LVI, and post-NACT Ki67 index (Table 2). Of these, clinical N2-3 stage, pathological T2-3 stage, pathological nodal positivity, triple-negative subtype, and post-NACT Ki67 $\geq 10 \%$ were independently associated with reduced DMFS in the multivariate analysis (Table 2). 
Table 2

Univariate and multivariate analyses for the risk factors associated with reduced distant metastasis-free survival

\begin{tabular}{|c|c|c|c|c|c|}
\hline \multirow{2}{*}{ Variables } & & \multicolumn{2}{|c|}{ Univariate analysis } & \multicolumn{2}{|c|}{ Multivariate analysis } \\
\hline & & $\mathrm{HR}(95 \% \mathrm{Cl})$ & $P$ & $\mathrm{HR}(95 \% \mathrm{Cl})$ & $P$ \\
\hline \multirow[t]{2}{*}{ Clinical T stage } & cT1-2 & Ref & 0.165 & & \\
\hline & сT3-4 & $\begin{array}{l}1.012(0.995- \\
1.029)\end{array}$ & & & \\
\hline \multirow[t]{2}{*}{ Clinical N stage } & $\mathrm{cNO}-1$ & Ref & $<.001$ & Ref & $\begin{array}{l}< \\
0.001\end{array}$ \\
\hline & $\mathrm{cN} 2-3$ & $\begin{array}{l}2.523(1.734- \\
3.671)\end{array}$ & & $\begin{array}{l}1.043(1.025- \\
1.061)\end{array}$ & \\
\hline \multirow[t]{2}{*}{ Pathological T stage } & урт0-1 & Ref & $<_{0.001}$ & Ref & 0.005 \\
\hline & урт2-3 & $\begin{array}{l}1.037(1.019- \\
1.056)\end{array}$ & & $\begin{array}{l}1.027(1.008- \\
1.047)\end{array}$ & \\
\hline \multirow[t]{2}{*}{$\begin{array}{l}\text { Pathological nodal } \\
\text { status }\end{array}$} & ypN- & Ref & $<.001$ & Ref & 0.005 \\
\hline & ypN+ & $\begin{array}{l}2.618(1.678- \\
4.086)\end{array}$ & & $\begin{array}{l}1.989(1.225- \\
3.229)\end{array}$ & \\
\hline \multirow[t]{2}{*}{ Molecular subtype } & $\begin{array}{l}\text { Non- } \\
\text { TNBC }\end{array}$ & Ref & $<.001$ & Ref & $\begin{array}{l}<.001 \\
0.001\end{array}$ \\
\hline & TNBC & $\begin{array}{l}2.584(1.706- \\
3.914)\end{array}$ & & $\begin{array}{l}3.137(1.996- \\
4.931)\end{array}$ & \\
\hline \multirow[t]{2}{*}{ pCR } & Yes & Ref & 0.006 & Ref & 0.333 \\
\hline & No & $\begin{array}{l}4.084(1.505- \\
11.080)\end{array}$ & & $\begin{array}{l}2.088(0.470- \\
9.271)\end{array}$ & \\
\hline \multirow[t]{2}{*}{ Histologic grade } & 1,2 & Ref & 0.149 & & \\
\hline & 3 & $\begin{array}{l}1.323(0.905- \\
1.933)\end{array}$ & & & \\
\hline \multirow[t]{2}{*}{ LVI } & No & Ref & $<.001$ & Ref & 0.313 \\
\hline & Yes & $\begin{array}{l}1.977(1.359- \\
2.874)\end{array}$ & & $\begin{array}{l}1.232(0.821- \\
1.848)\end{array}$ & \\
\hline
\end{tabular}

$\mathrm{CM}$, conventional mastectomy; HER2, human epidermal growth factor receptor 2; HR, hazard ratio; IBR, immediate breast reconstruction; IDC, invasive ductal carcinoma; ILC, invasive lobular carcinoma; LVI, lymphovascular invasion; NACT, neoadjuvant chemotherapy; pCR, pathological complete response; TNBC, triple-negative breast cancer. 


\begin{tabular}{|c|c|c|c|c|c|}
\hline \multirow[b]{2}{*}{ Post-NACT Ki67 } & \multirow[b]{2}{*}{$<10 \%$} & \multicolumn{2}{|c|}{ Univariate analysis } & \multicolumn{2}{|c|}{ Multivariate analysis } \\
\hline & & Ref & 0.009 & Ref & 0.048 \\
\hline & $\geq 10 \%$ & $\begin{array}{l}1.669(1.134- \\
2.457)\end{array}$ & & $\begin{array}{l}1.517(1.005- \\
2.290)\end{array}$ & \\
\hline \multicolumn{6}{|c|}{$\begin{array}{l}\text { CM, conventional mastectomy; HER2, human epidermal growth factor receptor 2; HR, hazard ratio; } \\
\text { IBR, immediate breast reconstruction; IDC, invasive ductal carcinoma; ILC, invasive lobular carcinoma, } \\
\text { LVI, lymphovascular invasion; NACT, neoadjuvant chemotherapy; pCR, pathological complete } \\
\text { response; TNBC, triple-negative breast cancer. }\end{array}$} \\
\hline
\end{tabular}

Patient, Tumor, and Treatment Characteristics for the Matched Groups

After propensity score matching, 228 patients were $1: 1$ matched into well-balanced IBR $(n=114)$ and CMalone $(n=114)$ groups. Patient and tumor characteristics for the matched groups are shown in Table 3. There were no significant differences between the matched groups in the selected matching variables (i.e., clinical T and $\mathrm{N}$ stages, molecular subtype, pCR status, and radiotherapy status) and pathological variables (pathological T and $\mathrm{N}$ stage, histotype, histologic grade, LVI, or post-NACT Ki67 status). The treatment characteristics for the matched groups are shown in Table 4. There were no significant differences between the matched groups in NACT regimens, adjuvant radiotherapy, adjuvant hormonal therapy, adjuvant chemotherapy, or targeted therapy for human epidermal growth factor 2-positive (HER2+) disease. 
Table 3

Patient and tumor characteristics for the matched groups

\begin{tabular}{|c|c|c|c|c|c|c|}
\hline \multicolumn{2}{|l|}{ Variables } & $\begin{array}{l}\text { IBR group, } n= \\
114\end{array}$ & $\%$ & $\begin{array}{l}\text { CM group, } \mathrm{n}= \\
114\end{array}$ & $\%$ & $P$ \\
\hline \multicolumn{2}{|l|}{$\begin{array}{l}\text { Age, Median (range), } \\
\text { years }\end{array}$} & \multicolumn{2}{|l|}{$35(23-40)$} & \multicolumn{3}{|l|}{$37(25-40)$} \\
\hline \multirow[t]{3}{*}{ Clinical T stage } & cT1 & 1 & 0.9 & 1 & 0.9 & 0.867 \\
\hline & cT2 & 53 & 46.5 & 49 & 43.0 & \\
\hline & cT3-4 & 60 & 52.6 & 64 & 56.1 & \\
\hline \multirow[t]{3}{*}{ Clinical N stage } & cNO & 21 & 18.4 & 21 & 18.4 & 0.839 \\
\hline & $\mathrm{cN} 1$ & 55 & 48.2 & 51 & 44.7 & \\
\hline & $\mathrm{cN} 2-3$ & 38 & 33.3 & 42 & 36.8 & \\
\hline \multirow[t]{4}{*}{ Molecular subtype } & HR+/HER2- & 47 & 41.2 & 49 & 43.0 & 0.940 \\
\hline & $\mathrm{HR}+/ \mathrm{HER} 2+$ & 30 & 26.3 & 29 & 25.4 & \\
\hline & HR-/HER2+ & 15 & 13.2 & 17 & 14.9 & \\
\hline & TNBC & 22 & 19.3 & 19 & 16.7 & \\
\hline \multirow[t]{2}{*}{$\mathrm{pCR}$} & Yes & 14 & 12.3 & 12 & 10.5 & 0.677 \\
\hline & No & 100 & 87.7 & 102 & 89.5 & \\
\hline \multirow[t]{3}{*}{ Pathological T stage } & ypTo/Tis & 16 & 14.0 & 16 & 14.0 & 0.578 \\
\hline & урT1 & 38 & 33.3 & 31 & 27.2 & \\
\hline & урт2-3 & 60 & 52.6 & 67 & 58.8 & \\
\hline \multirow[t]{3}{*}{ Pathological N stage } & ypNO & 40 & 35.1 & 41 & 36.0 & 0.906 \\
\hline & ypN1 & 39 & 34.2 & 41 & 36.0 & \\
\hline & ypN2-3 & 35 & 30.7 & 32 & 28.1 & \\
\hline \multirow[t]{3}{*}{ Histotype } & IDC & 104 & 91.2 & 97 & 85.1 & 0.536 \\
\hline & ILC & 2 & 1.8 & 3 & 2.6 & \\
\hline & Mixed & 4 & 3.5 & 6 & 5.3 & \\
\hline \multicolumn{7}{|c|}{$\begin{array}{l}\mathrm{CM} \text {, conventional mastectomy; HER2, human epidermal growth factor receptor 2; HR, hormone } \\
\text { receptor; IBR, immediate breast reconstruction; IDC, invasive ductal carcinoma; ILC, invasive lobular } \\
\text { carcinoma; LVI, Iymphovascular invasion; NACT, neoadjuvant chemotherapy; pCR, pathological } \\
\text { complete response; TNBC, triple-negative breast cancer. }\end{array}$} \\
\hline \multicolumn{7}{|c|}{$\begin{array}{l}\text { SSM, skin-sparing mastectomy; T, taxane; TEl, tissue expander insertion; TNBC, triple-negative breast } \\
\text { cancer. *Unknown, } n=21\end{array}$} \\
\hline
\end{tabular}




\begin{tabular}{|c|c|c|c|c|c|c|}
\hline \multirow[t]{2}{*}{ Variables } & & $\begin{array}{l}\text { IBR group, } n= \\
114\end{array}$ & $\%$ & $\begin{array}{l}\text { CM group, } n= \\
114\end{array}$ & $\%$ & $P$ \\
\hline & Others & 4 & 3.5 & 8 & 7.0 & \\
\hline \multirow[t]{2}{*}{ Histologic grade } & 1,2 & 69 & 60.5 & 76 & 66.7 & 0.335 \\
\hline & 3 & 45 & 39.5 & 38 & 33.3 & \\
\hline \multirow[t]{2}{*}{ LVI } & Yes & 55 & 48.2 & 46 & 40.4 & 0.230 \\
\hline & No & 59 & 51.8 & 68 & 59.6 & \\
\hline \multirow[t]{2}{*}{ Post-NACT Ki67 * } & $<10 \%$ & 50 & 49.5 & 50 & 47.2 & 0.737 \\
\hline & $\geq 10 \%$ & 51 & 50.5 & 56 & 52.8 & \\
\hline \multicolumn{7}{|c|}{$\begin{array}{l}\text { CM, conventional mastectomy; HER2, human epidermal growth factor receptor 2; HR, hormone } \\
\text { receptor; IBR, immediate breast reconstruction; IDC, invasive ductal carcinoma; ILC, invasive lobular } \\
\text { carcinoma; LVI, lymphovascular invasion; NACT, neoadjuvant chemotherapy; pCR, pathological } \\
\text { complete response; TNBC, triple-negative breast cancer. }\end{array}$} \\
\hline
\end{tabular}


Table 4

Treatment characteristics for the matched groups

\begin{tabular}{|c|c|c|c|c|c|c|}
\hline Variables & & $\begin{array}{l}\text { IBR group, } \mathrm{n}= \\
114\end{array}$ & $\%$ & $\begin{array}{l}\text { CM group, } n= \\
114\end{array}$ & $\%$ & $P$ \\
\hline \multirow[t]{3}{*}{ NACT regimen } & $A C+/-T$ & 101 & 88.6 & 101 & 88.6 & 0.328 \\
\hline & $\mathrm{T}$ & 9 & 7.9 & 12 & 10.5 & \\
\hline & Others & 4 & 3.5 & 1 & 0.9 & \\
\hline \multirow[t]{3}{*}{ Mastectomy type } & NSM & 78 & 68.4 & 0 & 0.0 & NA \\
\hline & SSM & 36 & 31.6 & 0 & 0.0 & \\
\hline & $\mathrm{CM}$ & 0 & 0.0 & 114 & 100.0 & \\
\hline \multirow[t]{2}{*}{$\begin{array}{l}\text { Reconstruction } \\
\text { Methods }\end{array}$} & $\begin{array}{l}\text { Autologous } \\
\text { flaps }\end{array}$ & 82 & 71.9 & 0 & 0.0 & NA \\
\hline & Implant & 32 & 28.1 & 0 & 0.0 & \\
\hline \multirow[t]{2}{*}{ Axillary surgery } & SLNB only & 48 & 42.1 & 34 & 29.8 & 0.053 \\
\hline & ALND & 66 & 57.9 & 80 & 70.2 & \\
\hline \multirow{2}{*}{$\begin{array}{l}\text { Adjuvant } \\
\text { radiotherapy }\end{array}$} & Yes & 91 & 79.8 & 87 & 76.3 & 0.522 \\
\hline & No & 23 & 20.2 & 27 & 23.7 & \\
\hline \multirow[t]{2}{*}{ Adjuvant HT } & Yes & 78 & 68.4 & 79 & 69.3 & 0.886 \\
\hline & No & 36 & 31.6 & 35 & 30.7 & \\
\hline \multirow[t]{2}{*}{ Adjuvant CTx } & Yes & 19 & 16.7 & 20 & 17.5 & 0.860 \\
\hline & No & 95 & 83.3 & 94 & 82.5 & \\
\hline \multirow{2}{*}{$\begin{array}{l}\text { Trastuzumab in } \\
\text { HER2+ }\end{array}$} & Yes & 45 & 100.0 & 45 & 97.8 & 1.000 \\
\hline & No & 0 & 0.0 & 1 & 2.2 & \\
\hline \multicolumn{7}{|c|}{$\begin{array}{l}\text { AC, anthracycline; ALND, axillary lymph node dissection; CM, conventional mastectomy; CTX, } \\
\text { chemotherapy; HER2, human epidermal growth factor receptor 2; HR, hormone receptor; HT, hormonal } \\
\text { therapy; IBR, immediate breast reconstruction; IDC, invasive ductal carcinoma; ILC, invasive lobular } \\
\text { carcinoma; MF/MC, multifocality/multicentricity; NA, not applicable; NACT, neoadjuvant } \\
\text { chemotherapy; NSM, nipple-sparing mastectomy; pCR, pathological complete response; SLNB, } \\
\text { sentinel lymph node biopsy; SSM, skin-sparing mastectomy; T, taxane; TEl, tissue expander insertion; } \\
\text { TNBC, triple-negative breast cancer }\end{array}$} \\
\hline
\end{tabular}

Oncologic Outcomes of the Matched Groups

After matching, the median follow-up periods were $73(21-128)$ and $78(9-138)$ months for the IBR and $\mathrm{CM}$-alone groups, respectively. There was no significant difference between the IBR and CM-alone groups 
in the rate of LRR as the first event (14\% vs. 15.8\%; $P=0.710)$; however, the IBR group was associated with significantly lower rates of DM (27.2\% vs. $40.4 \% ; P=0.036)$ and breast cancer-associated death (14.9\% vs. $27.2 \% ; P=0.023$ ) than the CM-alone group. There was no significant difference between the IBR and CM-alone groups in the 5-year LRFS (84.5\% vs. 83.8\%; $P=0.582$ ) rate (Fig. 1A); however, the IBR group was associated with significantly improved 5-year DMFS (74.1\% vs. 62.6\%; $P=0.043)$ rate (Fig. $1 \mathrm{C})$ and BCSS (89.1\% vs. 77.6\%; $P=0.048)$ rate (Fig. 1D). The IBR group was also associated with an improved 5-year DFS (67\% vs. 58.5\%; $P=0.091)$ rate with borderline statistical significance compared to the CM-alone group (Fig. 1B).

\section{Discussion}

To the best of our knowledge, this is the first report on the comparison of oncologic outcomes following NSM/SSM with IBR versus CM alone, specifically in young women with breast cancer who received NACT. In this study, we observed similar LRR rates between the IBR and CM-alone groups. Moreover, our results showed that NSM/SSM with IBR does not impair the breast cancer outcomes compared with CM alone; on the contrary, we observed that NSM/SSM with IBR was associated with significantly improved 5-year DMFS and BCSS rates compared to CM alone. Furthermore, we found that DM was the most frequently observed recurrence type in the current setting, and tumor features including clinical N2-3 stage, pathological T2-3 stage, pathological nodal positivity, triple-negative subtype, and post-NACT Ki67 $\geq$ $10 \%$ were independently associated with increased risk of DM.

Recently, there has been an increase in the number of breast cancer patients undergoing NSM/SSM with IBR after NACT [19]. Young women with breast cancer were associated with an increased likelihood of undergoing this procedure compared with their older counterparts [19, 23]. In this study, we also observed that postmastectomy IBR was performed more frequently in patients aged $\leq 40$ years than in those aged $>40$ years in the NACT setting $(64.3 \%$ vs. $32 \%$; $P<0.001)$. However, younger patients tend to present with more advanced tumor stage, higher histological grade, more LVI, and more aggressive molecular subtype (i.e., HER2-positive/triple negative) [8-10]. Conversely, several studies have reported that the rate of pCR following NACT, which is a surrogate marker for favorable prognosis, was significantly higher among younger patients than in older patients $[24,25]$. Accordingly, NACT may be beneficial for young breast cancer patients. However, fear of cancer recurrence can be an imperative reason for not undergoing postmastectomy breast reconstruction in this patient population [26]. There are limited recurrence and survival data available regarding IBR after NACT in young patients that can make it challenging for both surgeons and patients with respect to an informed decision-making process regarding surgical options.

Previous studies have reported improved prognosis in postmastectomy breast reconstruction compared with mastectomy alone for breast cancer [27-31]. In a study based on the data from the United States National Cancer Institute's Surveillance, Epidemiology, and End Results registries, Bezuhly et al. demonstrated that IBR is associated with improved BCSS compared to mastectomy alone, particularly among younger women (age < 50 years) with breast cancer [27]. In a retrospective study by Petit et al., the authors performed a matched cohort comparison of 146 patients who underwent implant-based breast 
reconstruction versus 146 patients who underwent mastectomy without reconstruction and found that the risks of DM and breast cancer-associated death were significantly lower in the breast reconstruction group than in the control group, while the LRR risk was similar between the matched groups [28]. In another matched cohort study by Eriksen et al., the DM rate and breast cancer mortality were significantly lower in the IBR group than in the mastectomy-alone group after a long-term follow-up [29]. Our findings that IBR is associated with lower rates of DM $(27.2 \%$ vs. $40.4 \%)$ and breast cancer mortality $(14.9 \%$ vs. $27.2 \%$ ) compared with $\mathrm{CM}$ alone are in agreement with these previously published studies [27-31]; however, our study differs from these previous studies in that our current analyses were exclusively performed in young patients (age $\leq 40$ years) receiving NACT, whereas the previous studies involved all age groups and did not compare the outcomes in young patient subgroups or in patients receiving NACT. There are generally substantial differences in baseline characteristics, oncological severity, and adjuvant therapy strategies between the patients undergoing postmastectomy breast reconstruction and those undergoing mastectomy alone. In our study, patients in the IBR group were more likely to be at earlier clinical stages and to have favorable molecular subtype (i.e., hormonal receptor-positive/HER2-negative), but less likely to undergo adjuvant radiotherapy than those in the $\mathrm{CM}$-alone group before matching. After propensity score-matching, these variables were well-balanced between the study groups. In addition, other tumor features including pathological stage, histotype, histologic grade, LVI, and post-NACT Ki67 status, as well as treatment characteristics including NACT regimens, adjuvant hormonal therapy, chemotherapy, and targeted therapy for HER2 + disease, were also identical between the matched groups. Nevertheless, the similarity in the results of our current study and of previously published studies [27-31] in terms of the improved prognosis in the IBR group may be a consequence of selection bias and other hidden confounders, such as socioeconomic status between the comparative groups, even though the main prognostic factors were matched. Another potential prognostic factor that deserves consideration, but was not taken into account in this study, is the stress-related psychosocial impact of the treatments. Successful postmastectomy breast reconstruction is associated with less mental distress and improved patient satisfaction, psychosocial well-being, and quality of life compared with mastectomy alone [3234]. On the contrary, patients who underwent mastectomy alone may have higher level of postoperative depression and anxiety [32]. Stress-related psychosocial factors may negatively influence the recurrence and survival outcomes of breast cancer $[35,36]$, and the young patients who have lost their breast due to breast cancer may have higher levels of emotional and psychosocial stress compared to their older counterparts [37].

Although the risk of LRR is generally considered to be an important concern in choosing postmastectomy breast reconstruction or not, there were no significant differences in LRR rate and LRFS between the $\mathrm{NSM} / \mathrm{SSM}$ with IBR group and CM-alone group in the current study. This result was consistent with those of previous studies investigating the LRR risk following breast reconstruction [28, 29, 38-40]. The most common type of recurrence in the current setting was DM with a rate of $29.9 \%$ for the entire cohort. Clinical $\mathrm{N}$ stage, pathological T stage, pathological nodal positivity, triple-negative subtype, and postNACT Ki67 index seemed to be the important risk factors associated with DMFS. The high rate of DM and 
poor prognosis in this study population highlights the need for better systemic treatment strategies, especially for young breast cancer patients with such risk factors.

This study is limited by its retrospective design, and the associated selection bias may exist even after matching the key clinical factors. Hidden confounding variables may include, but are not limited to, socioeconomic and psychosocial factors and other clinicopathologic factors. In addition, this study was from a single-institution and involved a relatively small number of patients. Further studies with larger sample size are needed to validate our results. However, in the absence of comparative data, our initial propensity score-matched analysis provides valuable data on the oncologic comparison of NSM/SSM with IBR versus $\mathrm{CM}$ alone and may help in the shared decision-making process for the high-risk patient population in the current setting.

\section{Conclusions}

Our results indicated that IBR with NSM/SSM did not negatively affect long-term oncologic outcomes compared to $\mathrm{CM}$ alone in young women with breast cancer receiving NACT. DM was the most frequently observed recurrence type and clinical $\mathrm{N}$ stage, pathological T stage, pathological nodal positivity, triplenegative subtype, and post-NACT Ki67 index were independently associated with reduced DMFS in the current setting.

\section{Declarations}

\section{Compliance with ethical standards}

Funding: None.

Conflict of interest: None.

Ethical approval: This study was approved by the institutional review board of Asan Medical Center, Seoul, Korea. All procedures performed in studies involving human participants were in accordance with the ethical standards of the institutional and/or national research committee and with the $1964 \mathrm{Helsinki}$ declaration and its later amendments or comparable ethical standards.

Informed consent: Because of the retrospective nature of the study, the requirement for informed consent was waived, and the study was conducted with the exemption of consent under IRB deliberation as it used a platform that offers unidentified clinical information for research purposes.

\section{References}

1. Sung H, Ferlay J, Siegel RL et al. Global cancer statistics 2020: GLOBOCAN estimates of incidence and mortality worldwide for 36 cancers in 185 countries [published online February 4, 2021]. $C A$ Cancer J Clin. doi:10.3322/caac.21660 
2. DeSantis CE, Ma J, Gaudet MM et al. Breast cancer statistics, 2019. CA Cancer J Clin. 2019;69(6):438-451. doi:10.3322/caac.21583

3. Copson E, Eccles B, Maishman T et al. Prospective observational study of breast cancer treatment outcomes for UK women aged 18-40 years at diagnosis: the POSH study. J Natl Cancer Inst. 2013;105(13):978-988. doi:10.1093/jnci/djt134

4. Kang SY, Kim YS, Kim Z et al. Breast cancer statistics in Korea in 2017: data from a breast cancer registry. J Breast Cancer. 2020;23(2):115-128. doi:10.4048/jbc.2020.23.e24

5. Lee SK, Kim SW, Yu JH et al. Is the high proportion of young age at breast cancer onset a unique feature of Asian breast cancer? Breast Cancer Res Treat. 2019;173(1):189-199. doi:10.1007/s10549-018-4947-z

6. Siegel RL, Miller KD, Fuchs HE, Jemal A. Cancer statistics, 2021. CA Cancer J Clin. 2021;71(1):7-33. doi:10.3322/caac. 21654

7. Narod SA. Breast cancer in young women. Nat Rev Clin Oncol. 2012;9(8):460-470. doi:10.1038/nrclinonc.2012.102

8. Kim J, Hong S, Lee JJ et al. Analysis of the tumor characteristics in young age breast cancer patients using collaborative stage data of the Korea central cancer registry [published online February 18, 2021]. Breast Cancer Res Treat. doi:10.1007/s10549-021-06107-9

9. Cathcart-Rake EJ, Ruddy KJ, Bleyer A, Johnson RH. Breast cancer in adolescent and young adult women under the age of 40 years [published online January 15, 2021]. JCO Oncol Pract. doi:10.1200/OP.20.00793

10. Hu X, Myers KS, Oluyemi ET, Philip M, Azizi A, Ambinder EB. Presentation and characteristics of breast cancer in young women under age 40. Breast Cancer Res Treat. 2021;186(1):209-217. doi:10.1007/s10549-020-06000-x

11. Avis NE, Crawford S, Manuel J. Quality of life among younger women with breast cancer. J Clin Oncol. 2005;23(15):3322-3330. doi:10.1200/JC0.2005.05.130

12. Rosenberg SM, Dominici LS, Gelber S et al. Association of breast cancer surgery with quality of life and psychosocial well-being in young breast cancer survivors. JAMA Surg. 2020;155(11):10351042. doi:10.1001/jamasurg.2020.3325

13. Golshan M, Loibl S, Wong SM et al. Breast conservation after neoadjuvant chemotherapy for triplenegative breast cancer: surgical results from the BrighTNess randomized clinical trial. JAMA Surg. 2020;155(3):e195410. doi:10.1001/jamasurg.2019.5410

14. Hoffman KE, Mittendorf EA, Buchholz TA. Optimising radiation treatment decisions for patients who receive neoadjuvant chemotherapy and mastectomy. Lancet Oncol. 2012;13(6):e270-276. doi:10.1016/S1470-2045(12)70038-4

15. Kim HJ, Dominici L, Rosenberg SM et al. Surgical treatment after neoadjuvant systemic therapy in young women with breast cancer: results from a prospective cohort study [published online December 23, 2020]. Ann Surg. doi:10.1097/SLA.0000000000004296 
16. Bailey CR, Ogbuagu O, Baltodano PA et al. Quality-of-life outcomes improve with nipple-sparing mastectomy and breast reconstruction. Plast Reconstr Surg. 2017;140(2):219-226. doi:10.1097/PRS.0000000000003505

17. Howard MA, Sisco M, Yao K et al. Patient satisfaction with nipple-sparing mastectomy: a prospective study of patient reported outcomes using the BREAST-Q. J Surg Oncol. 2016;114(4):416-422. doi:10.1002/jso.24364

18. Drucker-Zertuche M, Robles-Vidal C. A 7 year experience with immediate breast reconstruction after skin sparing mastectomy for cancer. Eur J Surg Oncol. 2007;33(2):140-146. doi:10.1016/j.ejso.2006.10.010

19. Wong SM, Chun YS, Sagara Y, Golshan M, Erdmann-Sager J. National patterns of breast reconstruction and nipple-sparing mastectomy for breast cancer, 2005-2015. Ann Surg Oncol. 2019;26(10):3194-3203. doi:10.1245/s10434-019-07554-x

20. Mandelbaum A, Nakhla M, Seo YJ et al. National trends and predictors of mastectomy with immediate breast reconstruction [ published online February 17, 2021]. Am J Surg. doi:10.1016/j.amjsurg.2021.02.014

21. Lazow SP, Riba L, Alapati A, James TA. Comparison of breast-conserving therapy vs mastectomy in women under age 40: national trends and potential survival implications. Breast J. 2019;25(4):578584. doi:10.1111/tbj.13293

22. Amin MB, Edge S, Greene F et al, eds. AJCC cancer staging manual. 8th ed. New York, NY: Springer; 2017

23. Christian N, Zabor EC, Cassidy M, Flynn J, Morrow M, Gemignani ML. Contralateral prophylactic mastectomy use after neoadjuvant chemotherapy. Ann Surg Oncol. 2020;27(3):743-749. doi:10.1245/s10434-019-08038-8

24. Loibl S, Jackisch C, Lederer B et al. Outcome after neoadjuvant chemotherapy in young breast cancer patients: a pooled analysis of individual patient data from eight prospectively randomized controlled trials. Breast Cancer Res Treat. 2015;152(2):377-387. doi:10.1007/s10549-015-3479-z

25. Villarreal-Garza C, Bargallo-Rocha JE, Soto-Perez-de-Celis E et al. Real-world outcomes in young women with breast cancer treated with neoadjuvant chemotherapy. Breast Cancer Res Treat 2016;157(2):385-394. doi:10.1007/s10549-016-3811-2

26. Nozawa K, Ichimura M, Oshima A et al. The present state and perception of young women with breast cancer towards breast reconstructive surgery. Int J Clin Oncol. 2015;20(2):324-331. doi:10.1007/s10147-014-0716-0

27. Bezuhly M, Temple C, Sigurdson LJ, Davis RB, Flowerdew G, Cook EF, Jr. Immediate postmastectomy reconstruction is associated with improved breast cancer-specific survival: evidence and new challenges from the surveillance, epidemiology, and end results database. Cancer. 2009;115(20):4648-4654. doi:10.1002/cncr.24511

28. Petit JY, Le MG, Mouriesse $\mathrm{H}$ et al. Can breast reconstruction with gel-filled silicone implants increase the risk of death and second primary cancer in patients treated by mastectomy for breast cancer? 
Plast Reconstr Surg. 1994;94(1):115-119. doi:10.1097/00006534-199407000-00011

29. Eriksen C, Frisell J, Wickman M, Lidbrink E, Krawiec K, Sandelin K. Immediate reconstruction with implants in women with invasive breast cancer does not affect oncological safety in a matched cohort study. Breast Cancer Res Treat. 2011;127(2):439-446. doi:10.1007/s10549-011-1437-y

30. Le GM, O'Malley CD, Glaser SL et al. Breast implants following mastectomy in women with earlystage breast cancer: prevalence and impact on survival. Breast Cancer Res. 2005;7(2):R184-193. doi:10.1186/bcr974

31. Platt J, Baxter NN, McLaughlin J, Semple JL. Does breast reconstruction after mastectomy for breast cancer affect overall survival: long-term follow-up of a retrospective population-based cohort. Plast Reconstr Surg. 2015;135(3):468e-476e. doi:10.1097/PRS.0000000000001054

32. Fanakidou I, Zyga S, Alikari V, Tsironi M, Stathoulis J, Theofilou P. Mental health, loneliness, and illness perception outcomes in quality of life among young breast cancer patients after mastectomy: the role of breast reconstruction. Qual Life Res. 2018;27(2):539-543. doi:10.1007/s11136-017-1735$\mathrm{x}$

33. Eltahir Y, Werners L, Dreise MM et al. Quality-of-life outcomes between mastectomy alone and breast reconstruction: comparison of patient-reported BREAST-Q and other health-related quality-of-life measures. Plast Reconstr Surg. 2013;132(2):201e-209e. doi:10.1097/PRS.0b013e31829586a7

34. Zehra S, Doyle F, Barry M, Walsh S, Kell MR. Health-related quality of life following breast reconstruction compared to total mastectomy and breast-conserving surgery among breast cancer survivors: a systematic review and meta-analysis. Breast Cancer (Auckl). 2020;27(4):534-566. doi:10.1007/s12282-020-01076-1

35. Chida Y, Hamer M, Wardle J, Steptoe A. Do stress-related psychosocial factors contribute to cancer incidence and survival? Nat Clin Pract Oncol. 2008;5(8):466-475. doi:10.1038/ncponc1134

36. Wang $X$, Wang N, Zhong $L$ et al. Prognostic value of depression and anxiety on breast cancer recurrence and mortality: a systematic review and meta-analysis of 282,203 patients. Mol Psychiatry. 2020;25(12):3186-3197. doi:10.1038/s41380-020-00865-6

37. Naik H, Leung B, Laskin J et al. Emotional distress and psychosocial needs in patients with breast cancer in British Columbia: younger versus older adults. Breast Cancer Res Treat. 2020;179(2):471477. doi:10.1007/s10549-019-05468-6

38. Wu ZY, Kim HJ, Lee JW et al. Long-term oncologic outcomes of immediate breast reconstruction vs conventional mastectomy alone for breast cancer in the setting of neoadjuvant chemotherapy. JAMA Surg. 2020;155(12):1142-1150. doi:10.1001/jamasurg.2020.4132

39. Lee SB, Lee JW, Son BH et al. Oncologic safety of skin-sparing mastectomy followed by immediate reconstruction in young patients with breast cancer. Asian J Surg. 2019;42(1):274-282. doi:10.1016/j.asjsur.2018.04.004

40. Zhang P, Li CZ, Wu CT et al (2017) Comparison of immediate breast reconstruction after mastectomy and mastectomy alone for breast cancer: a meta-analysis. Eur J Surg Oncol 43(2):285-293. doi:10.1016/j.ejso.2016.07.006 
Figures

A

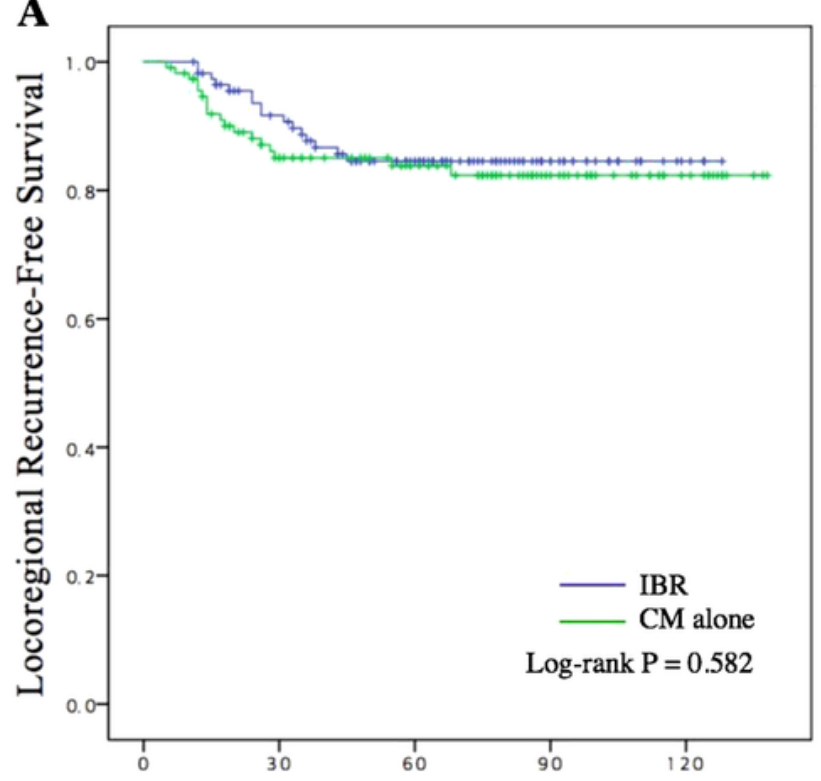

Time (months)

\begin{tabular}{rrrrrc} 
No. at risk & & \multicolumn{4}{c}{ Time (months) } \\
IBR & 114 & 94 & 62 & 25 & 5 \\
CM alone & 114 & 81 & 62 & 33 & 13
\end{tabular}

$\mathbf{C}$

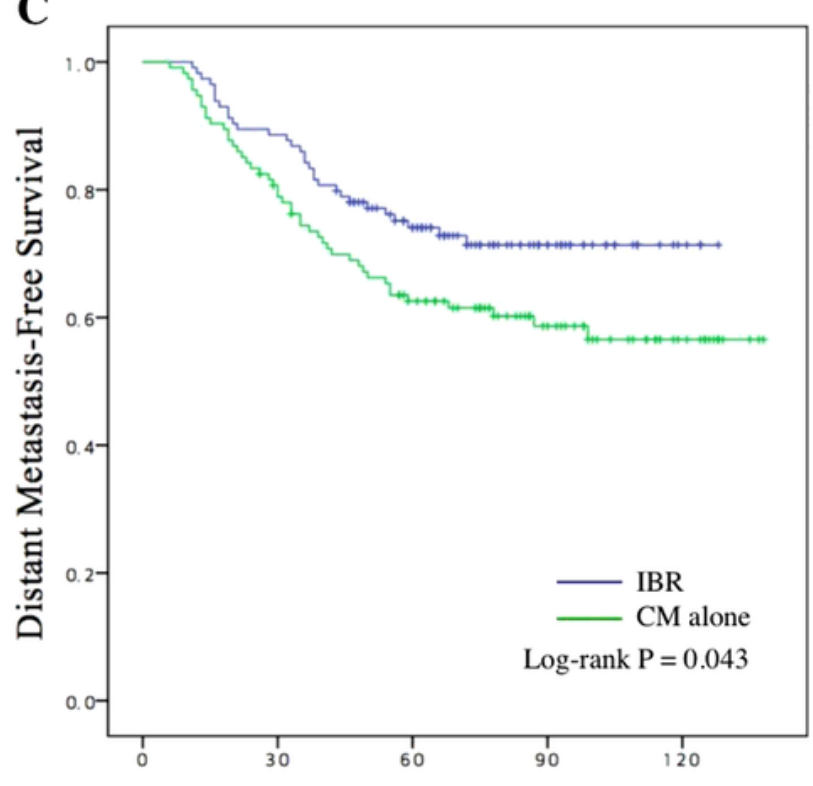

Time (months)

\begin{tabular}{rccccc} 
No. at risk & \multicolumn{5}{c}{ Time (months) } \\
IBR & 114 & 101 & 68 & 28 & 5 \\
CM alone & 114 & 88 & 65 & 35 & 13
\end{tabular}

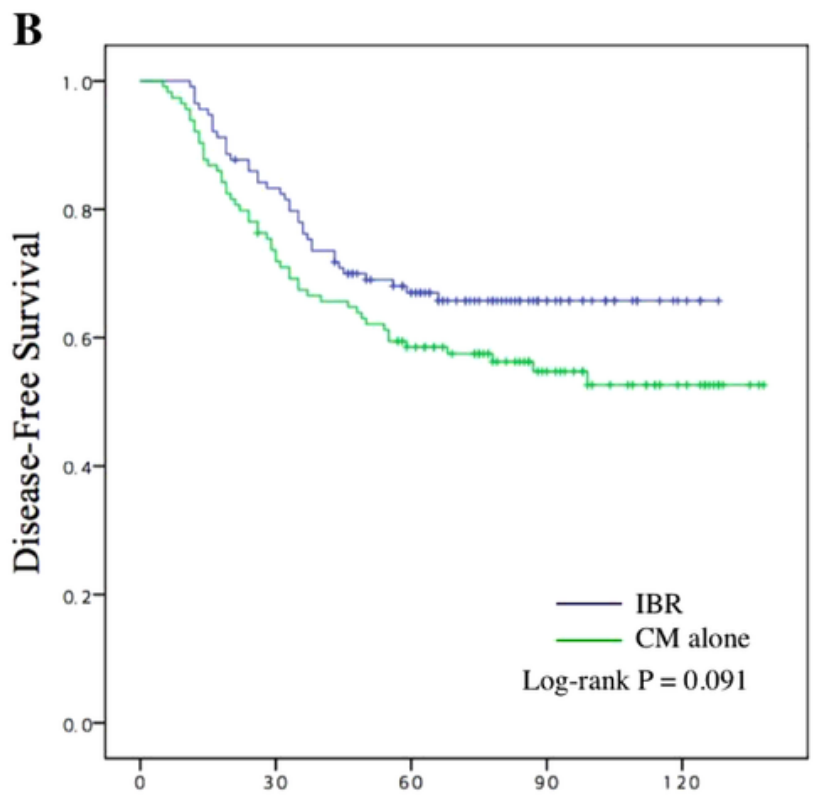

Time (months)

\begin{tabular}{rrrrrc} 
No. at risk & & \multicolumn{5}{c}{ Time (mo } \\
IBR & 114 & 94 & 62 & 25 & 5 \\
CM alone & 114 & 81 & 62 & 33 & 13
\end{tabular}

D

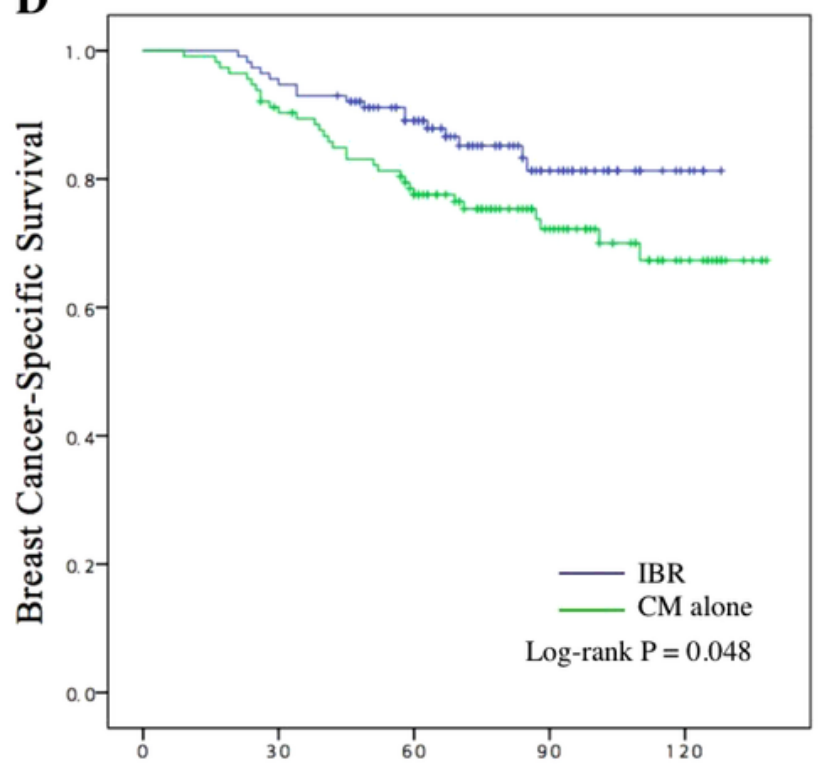

Time (months)

$\begin{array}{rrr}\text { No. at risk } & & \\ \text { IBR } & 114 & 108 \\ \text { CM alone } & 114 & 101\end{array}$

\section{Figure 1}

Propensity score-matched Kaplan-Meier survival analyses of locoregional recurrence-free (A), diseasefree (B), distant metastasis-free (C), and breast cancer-specific survival (D) between IBR and CM-alone groups. CM, conventional mastectomy; IBR, immediate breast reconstruction 